\title{
Simulative Investigations for Robust Frequency Estimation Technique in OFDM System
}

\author{
Kussum Bhagat $^{1}$ and Jyoteesh Malhotra ${ }^{2}$ \\ ${ }^{1}$ ECE Department, Guru Nanak Dev University Regional Campus, Jalandhar, \\ India \\ ${ }^{2}$ ECE Department, Guru Nanak Dev University Regional Campus, \\ Jalandhar, India \\ kussum.bhgt73991@gmail.com,jyoteesh@gmail.com
}

\begin{abstract}
OFDM (Orthogonal Frequency Division Multiplexing) is a promising technique for $4 G$ and 3GPP LTE system in terms of providing the higher data rate and efficient use of bandwidth. But OFDM suffers from ISI and ICI interferences in wireless and fading environment. There is a loss of certain amount of data because of these interferences, thus synchronization is required. It is important to estimate and correct the start of OFDM symbol efficiently to reduce timing and frequency offset errors. The paper mainly focus on the CFO problem present in OFDM system, describes the effect of CFO in OFDM system and provides the estimation techniques for CFO in time domain and frequency domain. The simulation results are performed for the CFO estimation technique with different values of $\mathrm{CFO}$ and then results has been compared.
\end{abstract}

Keywords: OFDM, CFO, ISI, ICI

\section{Introduction}

OFDM is a digital multicarrier modulation scheme which multiplexes the data on multiple carriers and transmits them in parallel. OFDM is very efficient technique; the reason behind this is OFDM uses orthogonal subcarriers which overlap in the frequency domain. Because of its orthogonal property the spectral efficiency is very high as compared to conventional frequency division multiplexing, which requires guard band between the adjacent sub-bands. The cyclic prefix (CP) or Guard band is required in OFDM. The purpose of CP is to remove the Inter-symbol interference (ISI) and channel effect. ISI can be avoided by inserting the $\mathrm{CP}$ with the length greater than the channel impulse response [1]. The drawback of cyclic prefix is that it is redundant and requires extra power to transmit along with the OFDM symbol whose effect appears in Signal to Noise Ratio (SNR). For the fast and accurate data transmission ISI and ICI (Inter Carrier Interference) arises in the transmission has to be eliminated as much as possible. ICI degrades the system performance but the feature of orthogonality provides the guarantee for the system to be ICI free. ICI can be removed by maintaining the orthogonality of carriers under the condition that the transmitter and the receiver have the exact same carrier frequency [2]. Thus OFDM is useful only when its orthogonality is maintained, if orthogonality removed, its performance will degrade due to ISI and ICI effects.

Synchronization has been become one of the important issue in OFDM system because of its sensitivity towards timing and frequency errors. There are two types of distortion associated with the carrier signal, symbol timing offset (STO) and carrier frequency offset (CFO). The local oscillators at the transmitter and receiver may not be synchronized and that results in STO and CFO. The symbol time offset is represented as 
phase rotation and inter-symbol interference. Carrier frequency offset is represented as phase rotation and inter channel interference [3].

Synchronization of an OFDM signal requires finding the symbol timing and carrier frequency offset. Finding the symbol timing for OFDM means finding an estimate of where the symbol starts. A symbol-timing synchronization must be performed to detect the starting point of each OFDM symbol. There is usually some tolerance for symbol timing errors when a cyclic prefix is used to extend the symbol. CFO is caused by Doppler spread, which is present in mobile systems. Due to changing channel conditions between the transmitter and the receiver, also contributes to the carrier offset. The CFO introduces ICI, which destroys the orthogonality among the subcarriers and attenuates the desired signal, reducing the effective Signal-to-Noise Ratio (SNR) .This results in degraded system performance [4].

The paper describes the effect of $\mathrm{CFO}$ and then discusses the synchronization techniques to remove the problem of CFO in OFDM system.

\section{Estimation Techniques for CFO}

As discuss above, to remove the effect of ISI and ICI due to STO and CFO estimation techniques are required.

\subsection{CFO Estimation Method}

CFO plays a key role in Frequency synchronization. Basically for getting a good performance of OFDM, the CFO should be estimated and compensated. Lack of the synchronization of the local oscillator signal for down conversion in the receiver with the carrier signal contained in the received signal causes CFO which causes Frequency mismatched in the transmitter and the receiver oscillator, ICI, Doppler Effect.

CFO estimation can be performed either in the time or the frequency domain. Frequency domain CFO estimation schemes can be further classified into two groups in the training symbol based approach and blind estimation approach. Moose in 1994 proposed a pilot based CFO estimation scheme by detecting the phase shifts between several successive identical pilot blocks. Schmidl and Cox in 1997 presented a robust synchronization scheme for OFDM using one unique OFDM symbol which has a repetition format within half a symbol period and the acquisition is achieved in two separate steps through the use of a two-symbol training sequence [5]. In [6] author discuss the training and blind algorithm. The design of the training sequence is such that is can lower the computational complexity. In blind CFO algorithm, by using the statistical properties of the received signal, the CFO will be estimated. Since the receiver doesn't have any knowledge of the signal that the transmitter has been sending, therefore the blind algorithms are considered to have a high computational complexity.

\section{System Methodology}

The section deals with the system methodology of estimation techniques for CFO. In this paper to estimate the $\mathrm{CFO}$ in time and frequency domain different methods are used. $\mathrm{CP}$ based method is used for the estimation of CFO in time domain and Moose and Classen method are used for the frequency domain. The modulation technique used here is QAM.

As the CFO cause the phase distortion in the OFDM symbol which describes the CFO effect. If channel effect is minimal and can be neglected then the phase difference of the $\mathrm{CP}$ and the OFDM symbol which is the victim of CFO can be found and the amount of $\mathrm{CFO}$ can be find by multiplying the OFDM symbol with $\mathrm{CP}$ and after that taking their 
phase angle measurements [7]. This is how the CFO can be estimated using CP based method but this method is only useful for the fractional CFO. Thus for the higher range of CFO Moose and Classen method is used. Moose gives the maximal like hood (ML) for carrier frequency offset estimation. In the Classen technique pilot tones can be inserted in the frequency domain and transmitted in every OFDM symbol for CFO tracking. After the synchronization symbol get extracted at the receiver side. Flow chart based on the $\mathrm{CFO}$ techniques which are used in this paper is given in fig. 1.

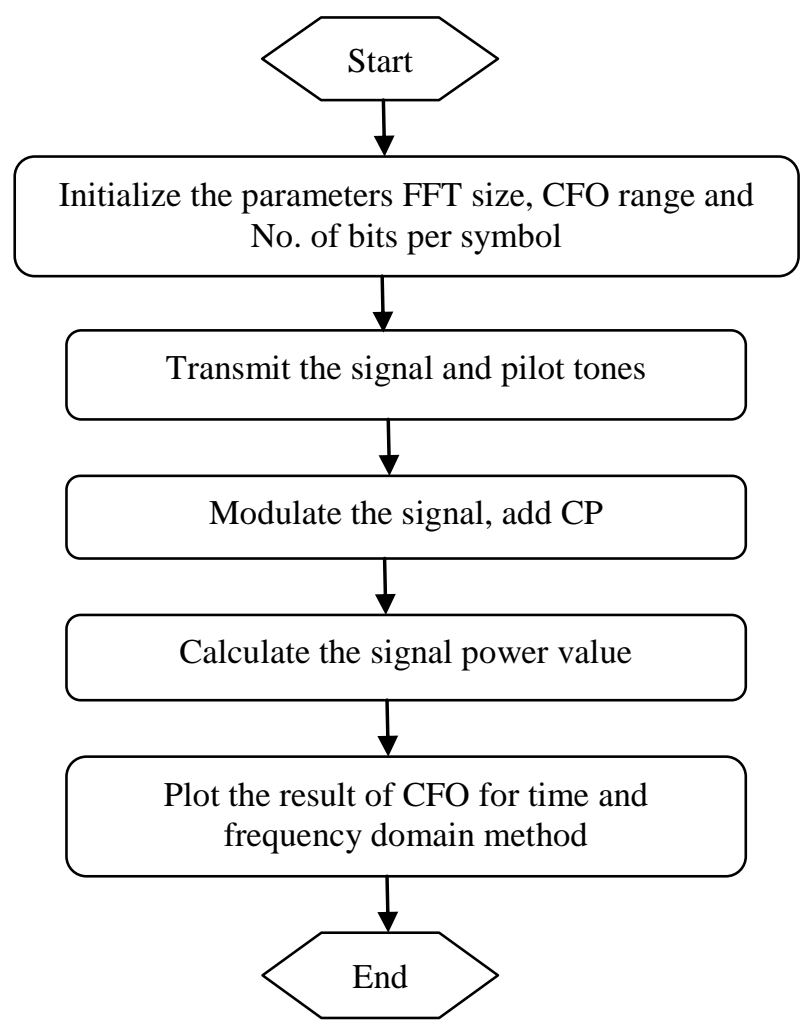

Figure 1.

Parameters which are used for the estimation for CFO in time and frequency domain is given in table 1 .

Table 1.

\begin{tabular}{|c|c|c|}
\hline S.No & Parameter & Value \\
\hline 1 & FFT size & 256 \\
\hline 2 & CFO range & 0.25 .0 .35 .0 .40 \\
\hline 3 & $\begin{array}{c}\text { No. of bits per } \\
\text { symbol }\end{array}$ & 4 \\
\hline
\end{tabular}




\section{Result and Discussion}

This section deals with the simulation results which are performed in Matlab for the estimation of CFO in time and frequency domain. The performance of $\mathrm{CP}$, Moose and Classen based techniques analyzed here. Figure 2(a), 2(b) and 2(c) shows the mean square error (MSE) performance with signal-to-noise ratio (SNR) in $\mathrm{dB}$. The simulation result compares the performance of CFO estimation with CFO range $=0.25,0.35$ and 0.40 . The simulation is done with FFT size 256 and number of bits per symbol is 4 .

Fig. 2 (a) plot the result for $\mathrm{CFO}=0.25$, shows that as the SNR of the received signal increases the mean square error decreases. Fig. shows the CFO estimation by using the phase difference between $\mathrm{CP}$ and corresponding part of OFDM symbol in case of $\mathrm{CP}$ based technique, by using the phase difference between two repetitive preambles in Moose method and using the phase difference between pilot tones in two OFDM symbols for Classen method. The performance of Classen method is better than CP and Moose based method.

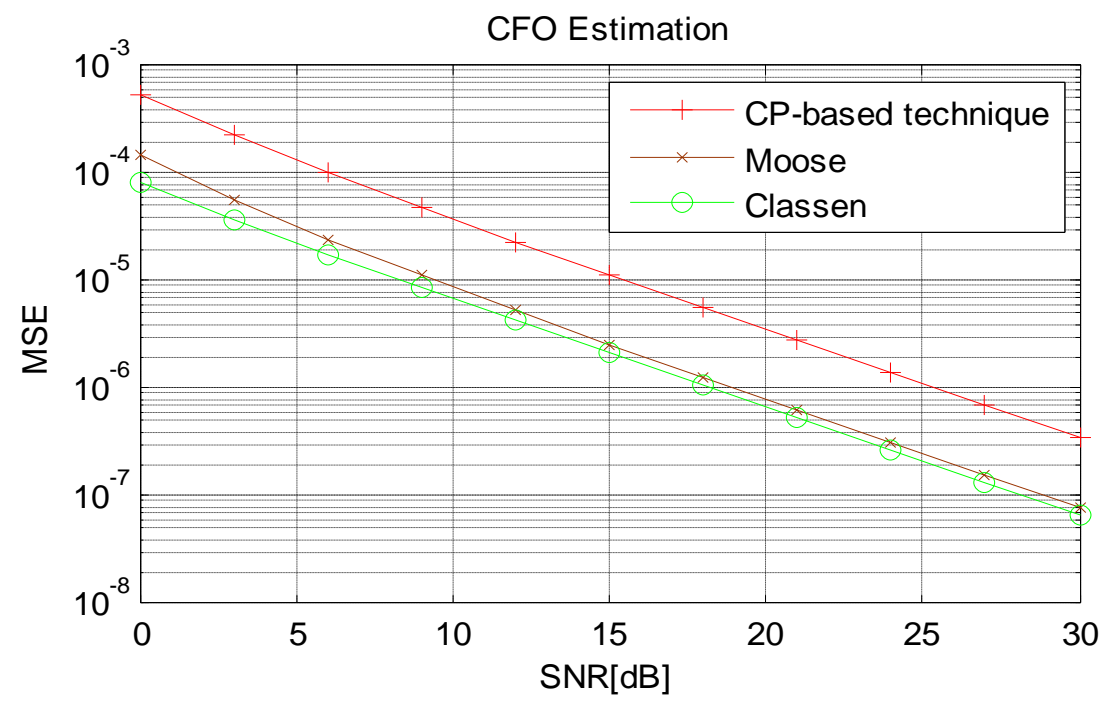

Figure 2(a). SNR Vs MSE

Performance of the estimation technique vary as the number of samples in $\mathrm{CP}$, number of samples in preamble and the no. of pilot tones vary. Fig. 2(b) and 2(c) shows the result for the CFO value 0.35 and 0.40 . Graph 2(b) reveals that as the CFO value increases from 0.25 to 0.35 the mean square error for the Classen method decreases. The effect of variation is more on the Classen method than $\mathrm{CP}$ based and Moose method. 


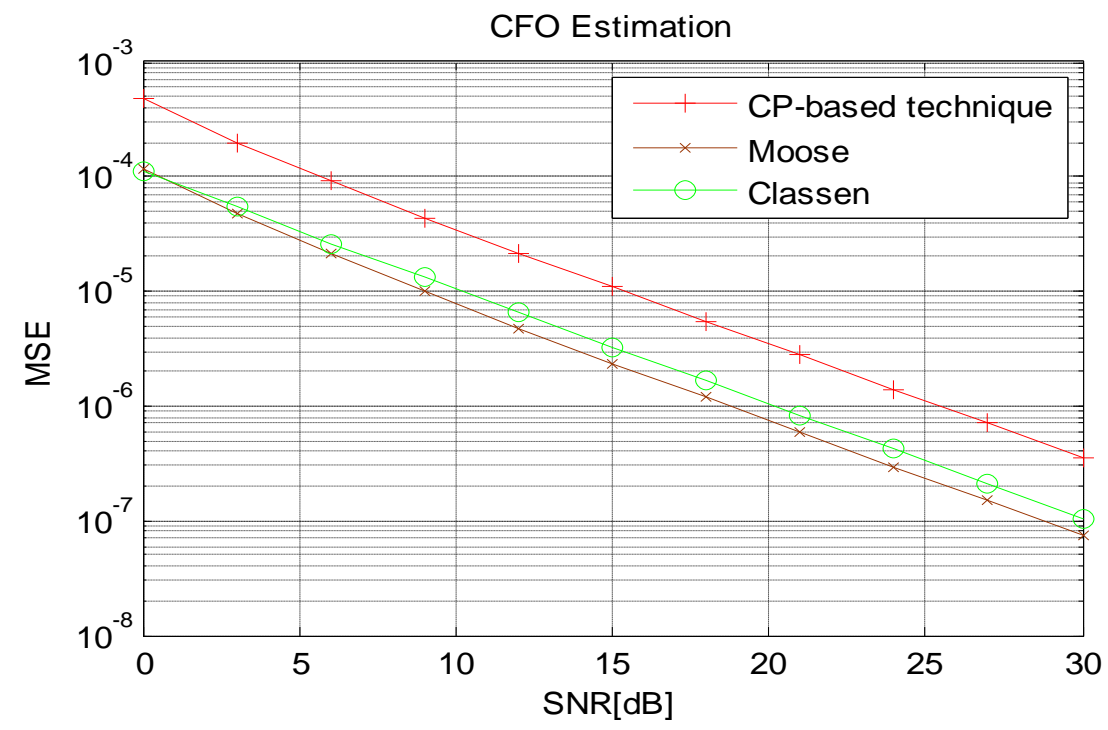

Figure 2(b). SNR Vs MSE

Fig. 2(c) plot the result for $\mathrm{CFO}=0.40$, the graph shows that there is no variation in MSE of Classen method with the increase of SNR. Thus the performance of Classen method degrades as the $\mathrm{CFO}$ value increases.

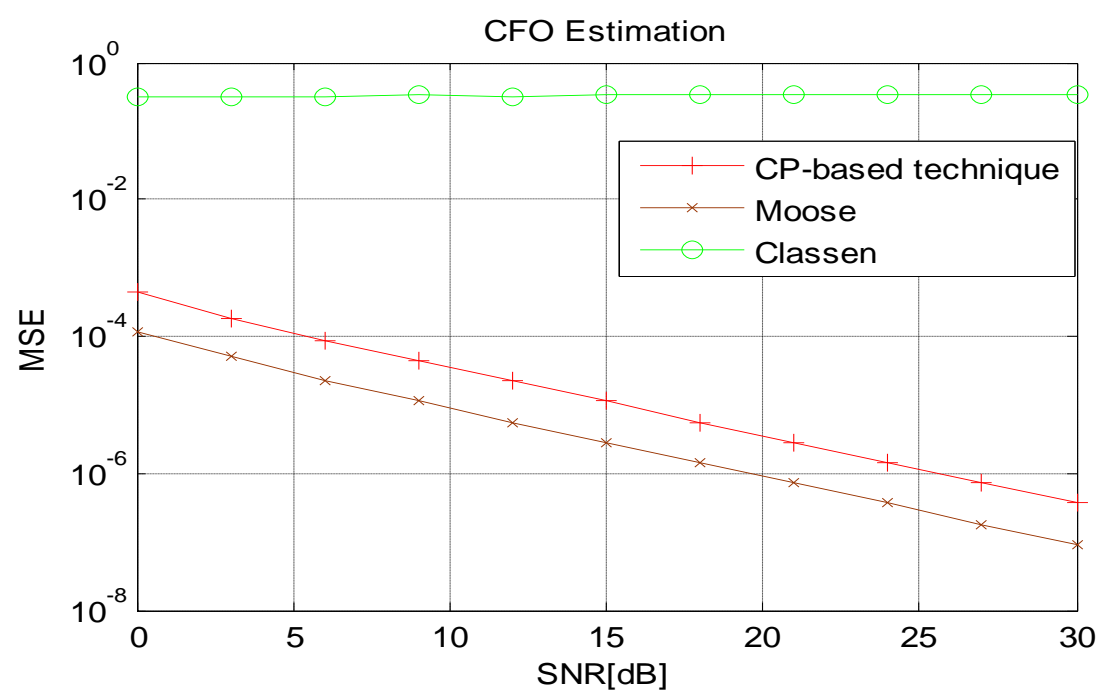

Figure 2(c). SNR Vs MSE

Table 2 analyzes the effect of MSE with respect to SNR for all the three techniques. The comparison shows that for the $\mathrm{CP}$ and Moose based method, when the value of CFO is changes from 0.25 to 0.35 there is no variation in signal to noise ratio and when the $\mathrm{CFO}=0.40$ then there is no variation in Classen method. Hence Classen method performs better for the lower $\mathrm{CFO}$ value and as the $\mathrm{CFO}$ increases its performance decreases. 
Table 2.

\begin{tabular}{|c|c|c|c|c|}
\hline MSE & CFO range & $\begin{array}{c}\text { CP based method } \\
(\text { SNR in dB) }\end{array}$ & $\begin{array}{c}\text { Moose } \\
\text { (SNR in dB) }\end{array}$ & $\begin{array}{c}\text { Classen } \\
\text { (SNR in dB) }\end{array}$ \\
\hline $10^{-6}$ & 0.25 & 25 & 18 & 17 \\
\hline $10^{-6}$ & 0.35 & 25 & 18 & 20 \\
\hline $10^{-6}$ & 0.40 & 25.5 & 19 & - \\
\hline
\end{tabular}

\section{Conclusion}

The main objective of this paper was to study the CFO effects and the need of synchronization in OFDM system. First the paper describes the problem of CFO in OFDM system and then gives the estimation techniques for CFO in time and frequency domain. The system methodology has been given for the CFO problem and then the simulation result has been analyzed. The simulation results for CFO estimation are obtained using $\mathrm{CP}$ based estimation and estimation technique proposed by Moose and Classen. The comparison of these three techniques shows that for the lower CFO range Classen method performs better as the SNR is $17 \mathrm{~dB}$ for MSE 10-6. But as the CFO increases Classen method shows no variation. Thus Classen method is superior for the less $\mathrm{CFO}$ range.

\section{References}

[1] M. Rao, M. Panchal and R. Jain, "ML Estimation of Initial Symbol Timing and Frequency Offset in OFDM Systems", International Journal of Engineering Research and Applications, vol. 2, no. 3, (2012).

[2] F. Wu and M. A. Abu-Rgheff, "Time and Frequency Synchronization Techniques for OFDM Systems operating in Gaussian and Fading Channels", Mobile Communication Network Research School of Computing, Communications \& Electronics University of Plymouth.

[3] D.-C. Chang, "Effect and Compensation of Symbol Timing Offset in OFDM Systems with Channel Interpolation" IEEE Transactions on Broadcasting, vol. 54, no. 4, (2008).

[4] I. Akbar and A. N. Malik, "Performance Analysis of Carrier Frequency Offset Estimation Techniques for OFDM Systems", Research Journal of Applied Science, Engineering and Technology Maxwell Scientific Organization, (2013).

[5] Y. S. Cho, J. Kim, W. Y. Yang and C. G. Kang, "MIMO-OFDM Wireless Communications with Matlab".

[6] S. Mohseni and M. A. Matin' "Study of the estimation techniques for the Carrier Frequency Offset (CFO) in OFDM systems", IJCSNS International Journal of Computer Science and Network Security, vol.12, no.6, (2012).

[7] W. Aziz, E. Ahmed, G. Abbas, S. Saleem and Q. Islam, "Performance Analysis of Carrier Frequency Offset (CFO) in OFDM using MATLAB", Journal of Engineering (JOE), vol. 1, no. 1, (2012). 